\title{
Social Innovation through Precision Farming: Leaf Disease Detection System Using IoT
}

\author{
Vijaysinh G. Chavan, Dipali C. Kothavale, Suryakant Patil
}

\begin{abstract}
Precision farming is a need of today's world. In last few decades image processing with IOT domain is rapidly increasing. In IOT domain we can easily use to multiple sensors for sensing unreadable format data. By using image processing domain we can easily work on complicated image to process efficiently. The plant disease detection is based on food security detection. The food security is based on food effect as well as environment. If food environment is not good then automatically food quality will loss. So we have to implement plant disease detection system using IOT and image processing. In this paper we have used four different sensors. 1. PH Sensor 2. Temperature sensor 3 . Humidity sensor 4. Soil Moisture Sensor. We can collect data through Raspberry pies well as collect plant images. The main goal of the proposed work is to monitor the plant leaf, detect and classify them according to the diseases using the data mining and image processing techniques. Geological condition is extraordinary for farming in light of the fact that it gives numerous good conditions. By collecting, the information from various types of sensors predicts the diseases that can affect the leaf. We have implemented the classification and clustering algorithm to sort out good quality and bad quality plant detection. Our segmentation approach and utilization of support vector machine demonstrate disease classification over 300images with an accuracy of $90 \%$.
\end{abstract}

Index Terms - Disease detection, Image processing, Precision farming, Pomegranate.

\section{INTRODUCTION}

The farmer cannot control the weather, if weather is changed then automatically affects on plant, which results in decreasing yield. Geological condition is uncertain for farming now days. So there is need to tackle it. Every plant suffers from $\mathrm{N}$ number of diseases, if uncertain changes occur in the climate. When plant suffers from some disease then automatically the production is decreases. Farmers used to monitor the plant at definite time intervals and if they are unable to identify the symptoms of a disease, they will apply

Manuscript revised on December 15, 2019 and published on December 27, 2019

Mr. Vijaysinh G. Chavan, Assistant Professor, CSE Department, SKN Sinhgad College of Engineering, Korti,Pandharpur. Research Scholar, Computer Science and Engg. Department, at Sandip University, Nashik, MH-India.

Ms. Dipali C. Kothavale, Has completed BE in CSE and is pursuing ME in CSE, SKN Sinhgad College of Engineering, Korti,Pandharpur.at Solapur University.

Dr. Suryakant Patil, working as a Professor and Dean (IPR and Consultancy), at Sandip University, Nashik, MH-India. approximate quantity of fertilizers or pesticides. But normally the farmers are not in a position to identify the actual disease deficiency. In last some decades the farmer cannot control this type of loss, because technology is not available to control plant disease due to uncertainty of weather. To motivate this point we have generated the IOT and image processing combine domain system, that detect the diseases on plant which occurs due to abnormal weather.

For the purpose of understanding we have taken the plant of grapes. At each stage of development of grapes we have seen different diseases. Many of the farmers can make illustration examination for detection and recognition of grape disease, also some of the systems use image processing method. This requires constant observing by specialists, which is tedious, costly and less precise. Therefore, farmers and experts want some quick some snappy, programmed, less costly exact strategy to identify disease infected on grapes. Automation of disease uncovering and observe can make easy under attack and timely disease manage which can lead to increased yield, improved crop excellence, and massive decrease in the quantity of applied pesticide.

As diseases leave some visible symptoms on the plants, particular on leaves, disease detection can be performed by imaging analysis of those visible patterns on leaves. Using digital image processing techniques, number of applications has found in different fields such as industrial inspection, medical imaging, remote sensing, and agricultural processing etc. For analysis in various agricultural applications, digital image processing techniques have been established as an effective way such as plant recognition, soil quality estimation, and crop yield estimation etc. in the field of agriculture. One of the applications of digital image processing techniques in agriculture is to detect plant disease.

\section{LITERATURE REVIEW}

Sabine D. Bauer Filip Korc described automatic classification methods on the high-resolution images. They performed operations on the sugar beet leaves to find the healthy and unhealthy parts of the leaves. Collected information fused into 3D model. Based on the Gaussian mixture by using the $\mathrm{k}$ nearest neighbor and naïve byes classification model based on pixels and achieved better results.Studied the conditional random field method for contextual classification of diseases [1].

Suyash S. Patil, Sandeep A. Thorat studied the diseases that on the grapes and causes of diseases. As per the producer's sprays maximum amount pesticides on the grapes, whichindirectly increases the production cost and also effects on the quality of grapes because sometimes farmers are not able to identify diseases. They proposed system to detect 
changes in the fruit and able to detect diseases using Hidden Morkov Model with the help of sensor and controllers [2]. Dheeb al Bashish, Malik Braik et.al aimed to implement a system that able to detect diseases automatically. Authors studied the real-time problem of farmers because of naked eye observation which is costlier and requires more time to find. They proposed image processing based solution based on RGB color transformation of images at first level. After that $\mathrm{k}$ - mean algorithm used to cluster the images and then texture of the image is calculated passed through a trained to find diseases. Experimental results show better accuracy [3].

A. Camargo, J.S. Smith studied image processing based diseases detection approach based on color classification into rgb H, I3a, I3b. These images then analyzed by histogram difference and threshold cut off [4].

Yin Laiwu1, Chen Deyun et.al.proposed A Recognition system on Optimal Wavelet Packet and Non-Negative Matrix Factorization for Extracting Pathologic Features of Plant Image. [5].

S. Arivazhagan, R. NewlinShebiah et.al presented a system that detects the unhealthy part of plant leaf using texture classification. In which diseased color transformation has done. Green pixels are masked on the images and then removed and applied segmentation process. Lastly, all texture is classified using classifier [6].

T. Rumpf, A.-K. Mahleinet. al. aimed to find non diseased leaf, differentiate according to diseases and Detect diseases based on symptoms. This is achieved by using the support vector machine with the help of kernel [7].

H. Al-Hiary, S. Bani-Ahmad et.al. present auto classification and detection system based on green pixels. In the first step, green coloured pixels are identified and then pixels are masked based on particular threshold values. Lastly pixels with rgb which contain zero are removed. Showed $83 \%$ accuracy in the detection [8].

X. F. Wang1, Z. Wang et.al.proposed data mining and fusion based solution with environmental information and apriority algorithm [9].

\section{PROPOSED SYSTEM}

In this, system provides us a solution for automatic detection and classification of plant leaf diseases and prediction using the Climatic Parameter Monitoring of Plants Using IoT. Detect the disease from the multilevel and hyper spectral images of plants which can be captured from the farm fields. It provides faster and more accurate solution embedded system, image process and wireless networking. At the initial step, the RGB images of all the leaf samples were picked up. Detection of infected part of disease is done and it contains two phases of detection. In this system, the framework for environmental parameter checking is implemented. By using the various types of sensors and collecting environmental information from them through the Controller, we can predict which type of disease will occur in the particular condition on the web page. Image processing segmentation is done on the image, which is based on edge detection and then image analysis. Images are captured and send to system in controlled environment are stored. Then Image segmentation separates the different parts or regions with special significance in the image, these regions do not cross each other. Image features such as boundary, gray scale conversion, shape, color and texture and all features are extracted for the disease spots to recognize diseases and recommendation about the diseases based on ecology climatically parameters. Our comprehensive control system measures reducing the occurrences of plant disease and ensure quality and accuracy of detection. Sensors are used to get environmental information and based on that information we can predict the chances to diseases.

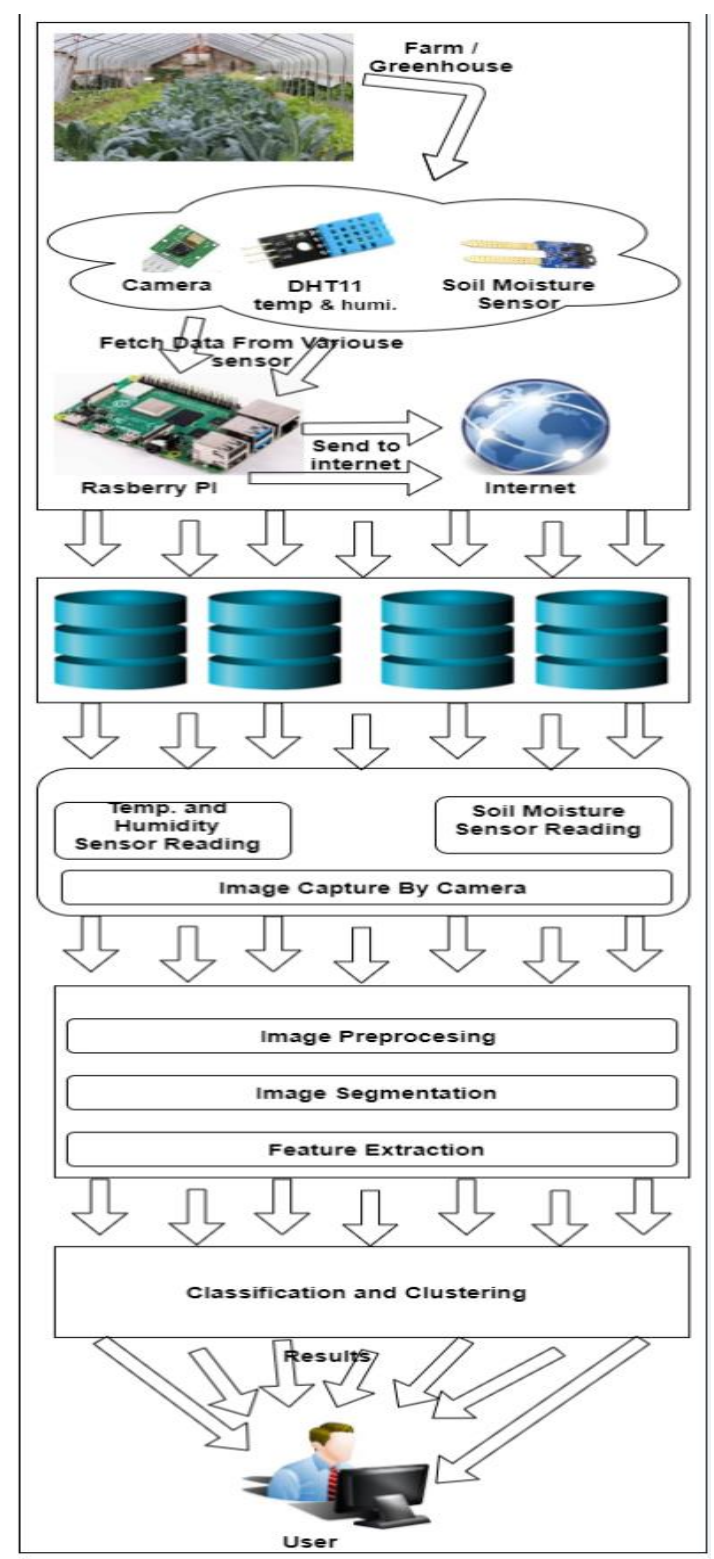

Fig.1. Proposed System

The above system architecture is consist of two parts first is the IoT part and second is software part. IoT part consist of Raspberry pi which is used to take the data from various sensors like DHT11 which gives current temperature and humidity reading, soil moisture sensor which gives moisture or water level present in soil and camera which gives images of leaves. All this data which is gathered by Raspberry pi is then send over internet which is stored in database for further processing and observation. 
In the second part i.e. software part, it shows the various sensors reading from the database on web page to user. To detect the disease on plants, images captured by user is given to the image processing module. Image processing operation is consist of image preprocessing which include gray scale conversion of images. After preprocessing image is passed to segmentation block which gives image pixel wise data and after that feature extraction is done on the images. After feature extraction classification and clustering is done on images which gives disease detected plant images.

\section{A. Methodologies Used:}

1. User Module: The first module in our project is the user module. User can register and login here.

2. Readings: In this module sensor data read from the sensor and shown on the web pages and store in the data base for the further processing. There are four sensor used in our system, Temperature sensor which is used to get the temperature from the root of the tree. Humidity sensor and moisture sensor gets the humidity and moisture from the roots. $\mathrm{pH}$ soil meter can be especially useful in lawn care in determining the soil conditions, this data is given to the micro-controller and all sensor readings show on the web pages.

3. Image Processing: All image processing operations are done in this module. The first phase in our system is the collection of the images of infected leaves. The images were getting from the system using the camera. Second phase is the processing of the images for the segmentation of the image which helps in the segmentation and analysis. Image is filtered to reduce the noise in the image. Noise occurs at the time of image acquisition. Next step is the segmentation, which plays important role in the detection. Segmentation is used to partition of the image to detect the infected part in the image. We use clustering algorithm to cluster images into number of parts. The next necessary step is to carry out the features extraction is. It consists of representing the segmented image on a vector of fixed features. They should be distinct and relevant for the classifier performance. The adopted features in this study include color, texture and shape. These features are used to find the exact shape of the defected image.

4. Classification: Analysis and classification of images. The classifier SI uses the colour to classify the images; it considers the diseases with similar or nearest colour, belonging to the same class. The sensor readings considers in this module. The remote measurement and controlling of different soil parameters along with leaf diseases detection over the Internet can be mechanized in this system. Images are then matched with dataset for diseases and find the top $\mathrm{k}$ nearest images. Finally disease and images shown in the ranking order are shown on the web page.

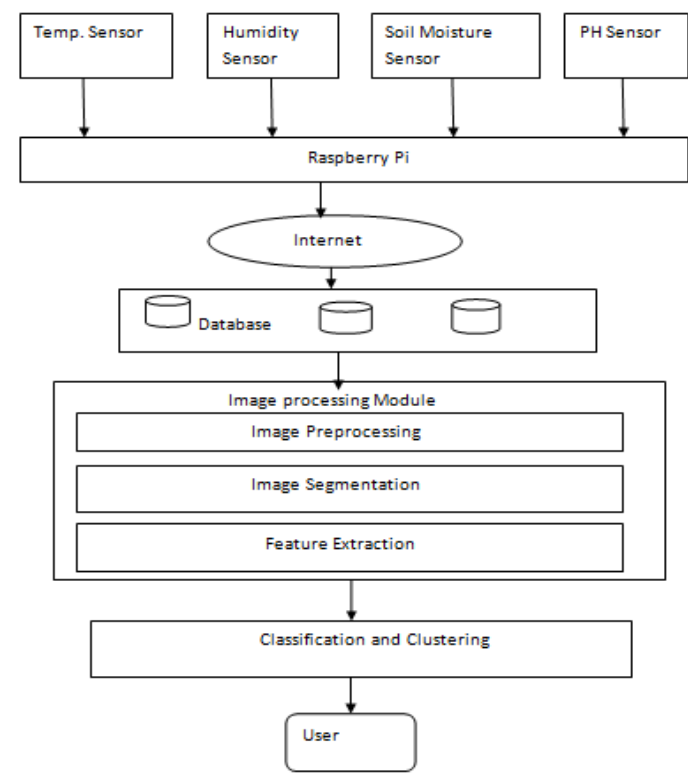

Fig. 2 Flow diagram

\section{B. Mathematical Module}

$\mathrm{S}$ is the set of sensor dataset of disease and the number of users. Sensor send the reading from the root of the plant eq. 1 shows the set of sensors. eq. 2 and 3 are the values and diseases data. $\mathrm{s}$ is the segmentation of the images of the plant leaf images

are segmented on the edge based or the region based. Values are stored in s then important features are extracted from the segmented parts through pattern recognition. Classifier $\mathrm{c}$ is applied on the image to classify parts these then compared with the disease name DN in the dataset to get final result or the type of the disease.

Let, $S=\{$ SEN $=\{$ Temps, Hums, PHs, Soils $\}$ RESEn , $\mathrm{U}=\{\mathrm{u} 1, \mathrm{u} 2, . . \mathrm{un}\}$,

$\mathrm{D}, \mathrm{s}, \mathrm{f}, \mathrm{c}, \mathrm{DN}, \mathrm{DD}, \mathrm{DN}, \mathrm{G}\}$ \}

$\mathrm{S}$ is set of sensor such as temperature, humidity, ph, soil moisture sensors start to read data from atmosphere.

Where,

SEN = Set of Sensors

Temps $=$ Temperature Sensor

HUMs = Humidity Sensor

$\mathrm{PHs}=\mathrm{PH}$ Sensor

Soils = Soil Sensor

RESEN $=$ Reading of Sensors

$\mathrm{F}(\mathrm{RESEN})=\int_{0}^{4}($ Temps $+\mathrm{HUMs}+\mathrm{PHs}+$ Soils $)---$

$\mathrm{D}=$ Dataset

$D=\sum_{0}^{n}$ (Values of Scientific results)

$\mathrm{U}=$ set of user

DN = Disease Name

$D N \in D \approx F(R E S E N)$

$\mathrm{DV}=$ Dataset Values

$\mathrm{s}=\{\mathrm{es}, \mathrm{rs}\}$ 
$\mathrm{s}=$ segmentation of image

es $=$ edge based segmentation

rs $=$ region based segmentation

$\mathrm{f}=$ feature extraction

$\mathrm{C}=$ Classifier

$\mathrm{F}(\mathrm{C})=\int_{0}^{\mathrm{m}}(\mathrm{s}+\mathrm{f}+\mathrm{DN})$-------------2

$\mathrm{DD}=$ Detection of Diseases

$\mathrm{DD} \in \mathrm{F}(\mathrm{RESEN}) \approx \mathrm{F}(\mathrm{C})$

\section{RESULT}

\section{A. Experimental Setup:}

Leaf disease detection system is implemented in JSP Servlet. It can be run on Windows XP/Windows Vista or on Windows 7 operating system or any other operating system. MySQL database is used to store data generated by the sensors and by user. To run web application locally we have used apache tomcat server. There are sensors required for the getting information.

\section{B. Experimental result:}

In graphical representation we are comparing to proposed system to existing approach. We have collected multiple images for experimentation. The leaf which has most green area then that plant is healthy and if any leaf has down to green area then we consider that the plant has a disease. In this system, we consider the Image colour combination data which is based on colour, RGB value. In RGB we calculate the Threshold value which can decide which portion of image is under disease. To identify colour combination in detail we create a clustering factor which is based on the HSV Colour conversion.

In colour conversion we can easily understand the difference between good image and disease image.

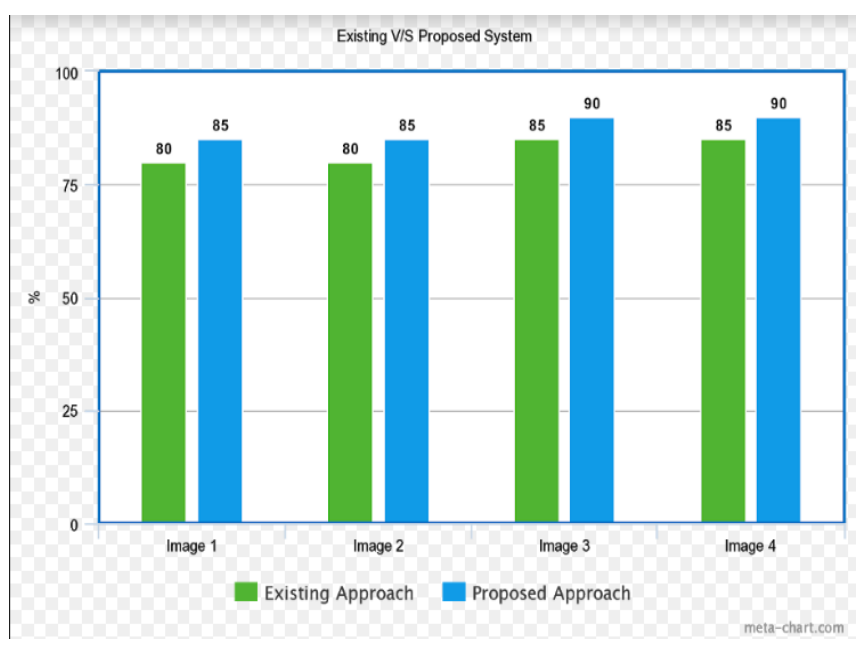

Fig. 3 Comparison of existing and proposed system
Table: 1. Result analysis

\begin{tabular}{|l|c|c|}
\hline Input & $\begin{array}{l}\text { Existing } \\
\text { Approach (in } \\
\% \text { ) }\end{array}$ & $\begin{array}{l}\text { Proposed Approach } \\
\text { (in \%) }\end{array}$ \\
\hline Image 1 & 80 & 85 \\
\hline Image 2 & 80 & 85 \\
\hline Image 3 & 85 & 90 \\
\hline Image 4 & 85 & 90 \\
\hline
\end{tabular}

Based on this we have analyzed that our system is enhanced and efficient than the earlier system.

\section{Conclusion}

A system we analyzed the climatically parametric condition and successfully tried to detect disease based on image processing techniques, and classify plant leaves using classifiers. By getting readings from sensor through controller predict the diseases that will occur on plant. Improved the disease detection accuracy, shows the efficiency of proposed algorithm in recognition and classification of the leaf diseases. This helps to the farmers to detect diseases and analyze large farm field's saves time and cost and increase the yield. In future we can try to reduce the ambiguity problem up to maximum extent. Sometime disease symptoms generates ambiguity problem. So we work on find out the specific disease as well as improve to detect or analyze all types of disease.

\section{ACKNOWLEDGMENT}

I would like to express my heartfelt gratitude to my guide Prof. Vijaysinh G. Chavan and who acted as a source of inspiration in all spheres of my dissertation phases and necessarily providing all resources along with a great platform to accomplish my target. I would like to give special thanks to our Head of Dept. Prof. Subhash V. Pingale and ME coordinator Prof. Namdev M. Sawant for giving valuable guidelines for completing this course.

\section{REFERENCES}

[1] Sabine D. Bauer FilipKorc Wolfgang Forstner, "The potential of automatic methods of classifications to identify leaf diseases from multispectral images", Published online : 26 January 2011Springer Science Business Media, LLC 2011.J. U. Duncombe, "Infrared navigation-Part I: An assessment of feasibility," IEEE Trans. Electron Devices, vol. ED-11, pp. 34-39, Jan. 1959.

[2] Suyash S. Patil, Sandeep A. Thorat,"Early Detection of Grapes Diseases Using Machine Learning and IoT",2016 Second International Conference on Cognitive Computing and Information Processing (CCIP).

[3] Dheeb Al Bashish, Malik Braik and Sulieman Bani Ahma,"Detection and classification of leaf diseases using k_means based segmentation and neural network based classification", vol:10, 2011.

[4] A. Camargo, J.S. Smith,"An image-processing based algorithm to automatically identify plant disease visual symptoms",BIOSYSTEMS ENGIMEERING 102 (2009) 9- 21

[5] Yin Laiwu1, Chen Deyun, Li Changcheng2 and Chen Dong,"'A Novel Recognition Algorithm Basedon Optimal Wavelet Packetand Non-Negative Matrix Factorization for Extracting Pathologic Features of Plant Image", Vol.6, No.5 (2013), pp.89-100.

[6] S.Arivazhagan, R. NewlinShebiah, S. Ananthi, S. Vishnu Varthini, "Detection of unhealthy region of plant leaves and classification of 


\section{Available online at www.ijrat.org}

plant leaf diseases using texture features", Vol. 15, No.1 211, March, 2013.R. A. Scholtz, "The Spread Spectrum Concept," in Multiple Access, N. Abramson, Ed. Piscataway, NJ: IEEE Press, 1993, ch. 3, pp. $121-123$.

[7] T. Rumpf, A.-K. Mahlein, et.al, "Early detection and classification of plant diseases with Support Vector Machines based on hyperspectral reflectance",Computers and Electronics in Agriculture 74 (2010) 91-99.

[8] H. Al-Hiary, S. Bani-Ahmad et.al,"Fast and Accurate Detection and Classification of Plant Diseases", International Journal of Computer Applications (0975 - 8887) Volume 17- No.1, March 2011.

[9] X. F. Wang, Z. Wang, S.W. Zhang, Y. Shi, "Monitoring and Discrimination of Plant Disease and Insect Pests based on agricultural IOT", International Conference on Information Technology and Management Innovation (ICITMI 2015).

[10] S.D.T. Kelly, N.K. Suryadevara and S.C. Mukhopadhyay, FIEEE, "Towards the Implementation of IoT for Environmental Condition Monitoring in Homes", 2013 IEEE.

\section{AUTHORS PROFILE}

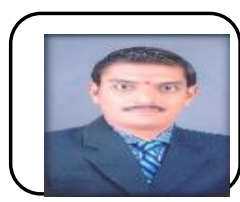

Mr. Vijaysinh G. Chavan, Assistant Professor, CSE Department,SKN Sinhgad College of Engineering, Korti,Pandharpur. Research Scholar, Computer Science and Engg. Department, at Sandip University, Nashik, MH-India. $\mathrm{He}$ has completed $\mathrm{BE}, \mathrm{ME}$ in $\mathrm{CSE}$ and is pursuing $\mathrm{PhD}$ in CSE. He is Research Scholar, at Sandip University, Nashik. He has 16 Research Papers in his portfolio. His research focused on IoT, Machine Learning.

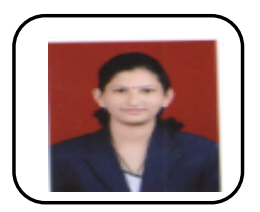

Ms. Dipali C. Kothavale, Has completed BE in CSE and is pursuing ME in CSE, SKN Sinhgad College of Engineering, Korti,Pandharpur.at Solapur University.

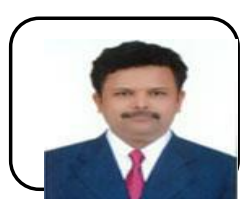

Dr. Suryakant Patil, working as a Professor and Dean (IPR and Consultancy), at Sandip University, Nashik, MH-India. Dr. Suryakant Patil has completed $\mathrm{BE}, \mathrm{ME}, \mathrm{PhD}$ in CSE. He is a Law Scholar with 107 complete patents and 86 Research Papers in his portfolio. He is Editorial Member as well as Reviewer of several International Journals /Conferences. His research focus on interdisciplinary Industrial and Social Innovation.
[11] Sanjay B. Patil, Dr. Shrikant K. Bodhe, "Leaf Disease Severity Measurement Using Image Processing", International Journal of Engineering and Technology Vol.3 (5), 2011, 297-301.

[12] S. Arivazhagan, R. NewlinShebiah*, S. Ananthi, S. Vishnu Varthini, "Detection of unhealthy region of plant leaves and classif ication of plant leaf diseases using texture features", AgricEngInt: CIGR Journal Open access at http://www.cigrjournal.org Vol. 15, No.1, pp.211-217, March 2013.

[13] Dheeb Al Bashish, Malik Braik, Suleiman Bani-Ahmed, "A framework for Detection and Classification of plant leaf and Stem Diseases,"20 10 international Conference on Signal and image processing, 2010, pp.113-118.

[14] Prof.Sanjay.B.Dhaygude, "Agricultural plant leaf disease detection using image processing.

[15] Lidi Wang, Tao Yang, Youwen Tian, "Crop Disease leaf image Segmentation Method Based on Colour Features, "The international Federation for information processing,. voI.258,2008,pp 713-717. 